\title{
Uso de esteróides anabolizantes em praticantes de musculação e/ou fisioculturismo
}

\author{
Clayton Luís Dornelles Macedo ${ }^{1}$, Rodrigo Pires dos Santos², Alessandro Comarú Pasqualotto², \\ Fábio Rogério Copette ${ }^{2}$, Sidnei Michel Pereira ${ }^{2}$, Alessandra Casagrande ${ }^{2}$, \\ Deluana Cunha Moletta ${ }^{2}$, Jiovani Fuzer ${ }^{2}$ e Sergio Augusto Veiga Lopes ${ }^{2}$
}

\begin{abstract}
RESUMO
Os hormônios andrógeno-anabolizantes têm seu uso difundido entre os esportistas, usualmente para aumentar a massa e a potência musculares. Assim, através do uso de questionários auto-aplicáveis em um estudo transversal, foram entrevistados 305 atletas (escolhidos aleatoriamente) das academias de Santa Maria, RS, a fim de quantificar diversas variáveis relacionadas ao uso de esteróides anabolizantes (EA). A maioria dos participantes era de homens $(81,1 \%)$ e a média de idade foi de 22 anos (DP \pm 7 ). Enquanto 29\% usavam aditivos alimentares, apenas $2 \%$ dos indivíduos admitiram ter usado EA (apesar de $46 \%$ dos entrevistados conhecerem algum usuário de EA). Dois terços dos usuários adquiriram a droga em farmácias (sem prescrição médica) e $35,1 \%$ desconheciam seus paraefeitos. Dos entrevistados, $28,4 \%$ acreditam que doses "adequadas" de EA não causam dano à saúde. Dessa forma, conclui-se que a principal medida preventiva à iniciação ao uso de EA é a educação dos atletas.
\end{abstract}

\section{ABSTRACT}

The use of anabolic-androgenic steroids by weight lifters and physical fitness athletes

Anabolic androgenic steroids are highly used among athletes, usually to increase muscle mass and strength. Therefore, using a self applicable questionnaire in a transversal study, 305 athletes were interviewed (randomically selected)

1. Professor Adjunto do Departamento de Clínica Médica da Universidade Federal de Santa Maria; Especialista em Medicina Esportiva e Doutor em Endocrinologia pela Escola Paulista de Medicina.

2. Acadêmico do Curso de Medicina da Universidade Federal de Santa Maria, RS.

Endereço para correspondência:

Universidade Federal de Santa Maria

Departamento de Clínica Médica

Avenida Roraima, Bairro Camobi

Santa Maria, RS from the Santa Maria physical fitness clubs, in order to establish many variables related to the use of anabolic steroids. Most participants were men $(81.1 \%)$ and mean age was 22 years (sd \pm 7 ). Although $29 \%$ used food additives, only $2 \%$ of the individuals admitted having used anabolic steroids (although 46\% of the individuals submitted to the questionnaire know a anabolic steroid user). Two-thirds of the users purchased the drug in drugstores (with no medical prescription) and $35.1 \%$ did not know the effects of the drug. Twenty-eight point four percent of the subjects believe that "adequate" doses of anabolic steroids are not harmful to health. Thus, the authors concluded that the main preventive step against the use anabolic steroids is to inform the athletes.

\section{INTRODUÇÃO}

Ao contrário dos hormônios femininos, estrógeno e progesterona, que têm sido amplamente prescritos por médicos e seus riscos e benefícios extensamente estudados, as preparações de andrógenos não são familiares à maioria dos clínicos e seus riscos e benefícios têm recebido pouca atenção ${ }^{1}$.

Os esteróides anabolizantes (EA) são derivados sintéticos da testosterona. A maioria dos esteróides sintéticos foi desenvolvida na década de 50, na tentativa de separar o hormônio masculinizante (andrógeno) de seus efeitos anabólicos ${ }^{2,3}$. Embora tenham indicações médicas precisas, os EA são freqüentemente usados para outras finalidades, amiúde na prática esportiva.

As indicações terapêuticas dos EA incluem hipogonadismo em adultos, certos tipos de anemia, anabolismo protéico, angioedema hereditário e certas condições ginecológicas. Têm também papel quando usados em déficit de crescimento e no tratamento da osteoporose $e^{4,5}$. Já em atletas, o padrão de uso de drogas é, sem dúvida, parte de um dinâmico e extremamente complexo processo relacionado a variáveis sociais, fisiológicas, psicológicas, econômicas e políticas. Embora o uso de EA por atletas alcance ênfase na mídia, geralmente em casos de doping ocorridos em grandes competições esportivas, todos os dados deixam claro que o abuso dessas drogas não é restrito aos atletas de elite ${ }^{6}$. 


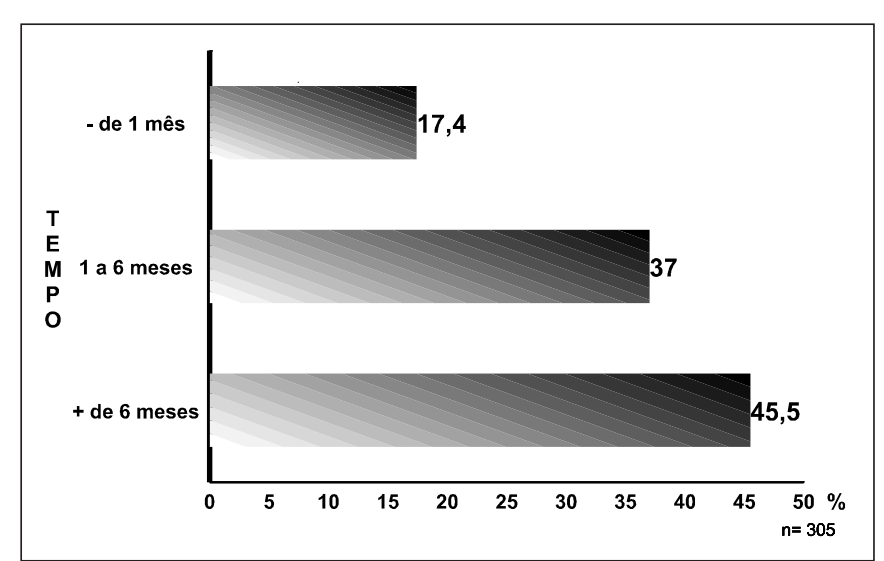

Fig. 1 - Distribuição dos praticantes de musculação elou fisioculturismo de acordo com o tempo de prática do esporte

Nos anos 50, surgiram na literatura os primeiros relatos de abuso de EA para fins não-terapêuticos, ocorridos em atletas russos de ambos os sexos ${ }^{7}$. Desde então, os hormônios andrógeno-anabolizantes vêm sendo usados, usualmente em pessoas com boas condições de saúde, para aumentar a massa e a potência musculares, além de "melhorar" a aparência física, a auto-estima e a capacidade de treinar em ritmo intenso $^{8-10}$. Seu uso para essa finalidade vem sendo condenado por muitas organizações, incluindo a American Medical Association e o American College of Sports Medicine, devido a seus conhecidos efeitos colaterais ${ }^{11}$.

De modo geral, os efeitos adversos do abuso de EA podem ser amplamente divididos em quatro categorias: 1) efeitos nas características sexuais secundárias e funções hormonais relatadas; 2) efeitos em tecidos somáticos, incluindo trombogênese e produção tumoral; 3) efeitos relacionados à adulteração e administração da droga, incluindo doenças infecciosas, especificamente hepatite B e HIV; e 4) efeitos no comportamento e saúde mental, incluindo o desenvolvimento de dependência. Numerosos artigos e uma variedade de textos têm abordado esses aspectos com detalhes ${ }^{7,12-19}$.

Apenas nos EUA, existem mais de 45 produtos contendo EA disponíveis no mercado ${ }^{20}$. A FDA (Food and Drug Administration) estima que 1 a 3 milhões de pessoas nos EUA usem essas drogas por razões não medicamentosas, alimentando um mercado negro que, por estimativa, excede os 300 milhões de dólares por ano, $3,6,21-23$. No Brasil, o uso de EA para fins não medicamentosos também parece ser bastante difundido, embora os dados a respeito de sua prevalência na literatura sejam escassos.

Dessa forma, os autores buscam, por meio deste trabalho, quantificar o número de usuários de tais substâncias nas academias de Santa Maria, RS, tornar evidentes os efeitos colaterais que essas drogas provocam, determinar o nível de informação a seu respeito e buscar meios educativos para a prevenção e controle do uso abusivo de EA em nossa região.

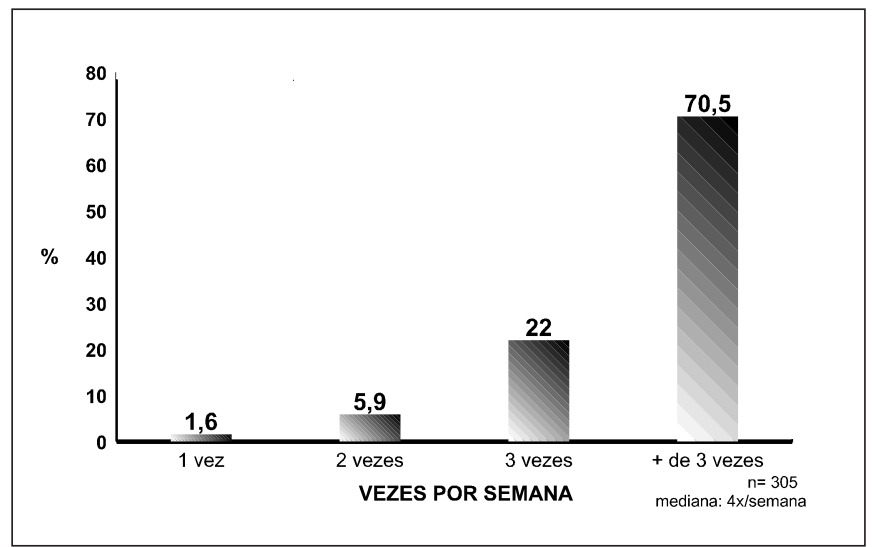

Fig. 2 - Distribuição dos praticantes de musculação elou fisioculturismo de acordo com a freqüência de comparecimento à academia

\section{MATERIAL E MÉTODOS}

Os autores realizaram um estudo transversal, sendo a amostra constituída aleatoriamente por praticantes de musculação e/ou fisioculturismo das seis maiores academias esportivas da cidade de Santa Maria, RS. Todas as instituições envolvidas forneceram aceite prévio à participação no projeto. Para inclusão no estudo, não houve restrição quanto a sexo ou idade, nem tampouco necessidade de identificação. Inicialmente, foi empregado um questionário auto-aplicado contendo, em sua maioria, questões fechadas. O questionário continha 26 questões e envolvia variáveis como sexo, idade, escolaridade e freqüência e tempo de prática de musculação e/ou fisioculturismo. Aos entrevistados foi perguntado, ainda, se estes já haviam ouvido falar a respeito de EA (e através de que meio), se conheciam algum usuário, quais os efeitos colaterais provocados pelo uso de EA e se já utilizaram aditivos alimentares ou mesmo EA. Para aqueles que se colocavam como usuários de EA, questionou-se onde iniciaram o uso, o tempo de uso, qual a principal razão para a utilização, a forma de aquisição, a via de administração, o esquema de utilização, o gasto mensal com o tratamento e quais as diferenças percebidas após a utilização. Ainda, foi oferecida aos usuários interessados uma série de exames laboratoriais gratuitos, mediante sua identificação para posterior contato. Após o preenchimento, os questionários foram depositados em urnas lacradas, abertas somente no momento da codificação dos dados. Após exclusão dos questionários com inconsistências importantes, os dados foram digitados e analisados com o auxílio do programa Epi-Info 6.0. Para evitar erros, foram realizadas duas digitações paralelas.

\section{RESULTADOS}

A amostra foi constituída de 305 praticantes de musculação e/ou fisioculturismo, distribuídos nas seis academias de maior fluxo da cidade de Santa Maria. A maioria dos partici- 


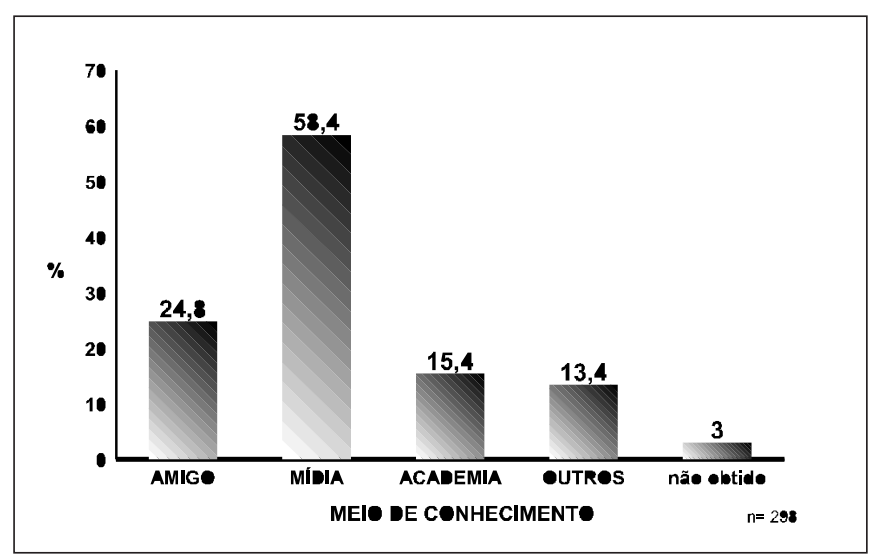

Fig. 3 - Distribuição dos praticantes de musculação elou fisioculturismo de acordo com o meio veiculador de informações sobre EA

pantes do estudo foi do sexo masculino $(81,1 \%)$. A média de idade foi de 22 anos (DP \pm 7 ); 78\% dos entrevistados encontravam-se na faixa dos 15 aos 24 anos de idade. Quanto ao grau de escolaridade, $9,2 \%$ tinham o $1 \stackrel{\circ}{\text { grau completo ou em }}$ curso, $49,5 \%$ o 2 o grau completo ou em curso e $29,5 \%$ o 3 은 grau (dado não obtido em $11,8 \%$ ).

As figuras 1 e 2 mostram, respectivamente, a distribuição da amostra de acordo com o tempo de prática do esporte e com a freqüência de comparecimento à academia. Quando interrogados quanto ao uso de aditivo alimentar, 29\% confirmaram já o terem feito uso alguma vez. O tipo de aditivo alimentar mais comumente usado por esses indivíduos foi vitaminas $(51,6 \%)$, seguido de aminoácidos $(50,5 \%)$ e repositivos hidreletrolíticos $(7,7 \%)$. Dezessete por cento afirmaram fazer uso de outros aditivos alimentares e 4,4\% não responderam à questão.

Duzentos e noventa e oito entrevistados $(97,7 \%)$ afirmaram já ter ouvido falar em EA. A figura 3 mostra os principais instrumentos veiculadores desse tipo de informação. Quando questionados sobre sua opinião a respeito do uso de EA e do possível surgimento de efeitos colaterais dele decorrentes, $71,6 \%$ ( $p<0,0001$, teste de duas proporções) entendem que, em qualquer dosagem, causa dano à saúde. Dezoito por cento afirmaram que doses adequadas não têm efeitos tóxicos e 10,4\%, que apenas com doses abusivas esses efeitos se tornam evidentes. Dos entrevistados, $46,2 \%$ referiram conhecer algum atleta usuário de EA. Com relação ao conhecimento dos possíveis paraefeitos advindos do uso de EA, 64,9\% afirmaram conhecê-los. Os efeitos colaterais mais citados por esses atletas estão descritos na figura 4.

Apenas $2 \%$ da amostra $(n=6)$ identificaram-se como usuários de EA. A figura 5 mostra as principais fontes que estimularam esses atletas ao uso; nenhum deles alegou indicação médica para tal prática. O tempo de uso de EA variou de 6 a 20 anos $(6,1 \pm 9,3$, média \pm DP), sendo este dado não

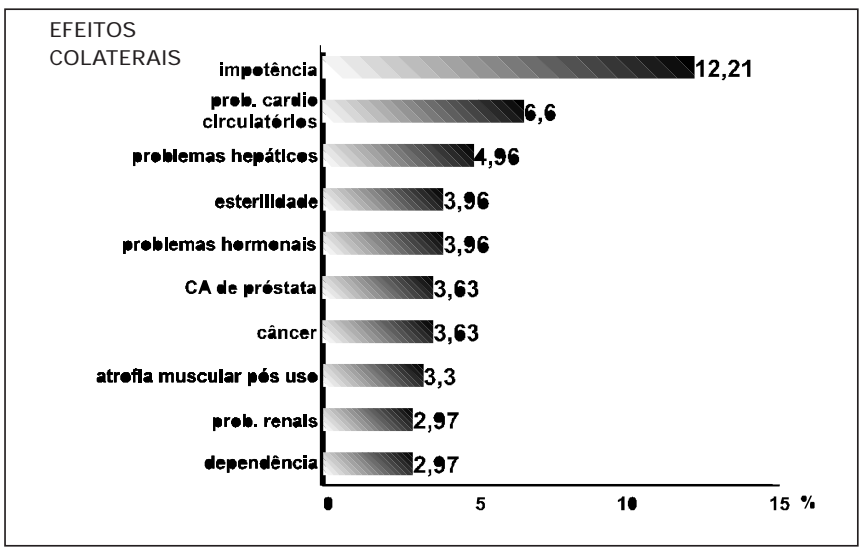

Fig. 4 - Distribuição dos praticantes de musculação elou fisioculturismo de acordo com os efeitos colaterais decorrentes do uso de EA mais citados

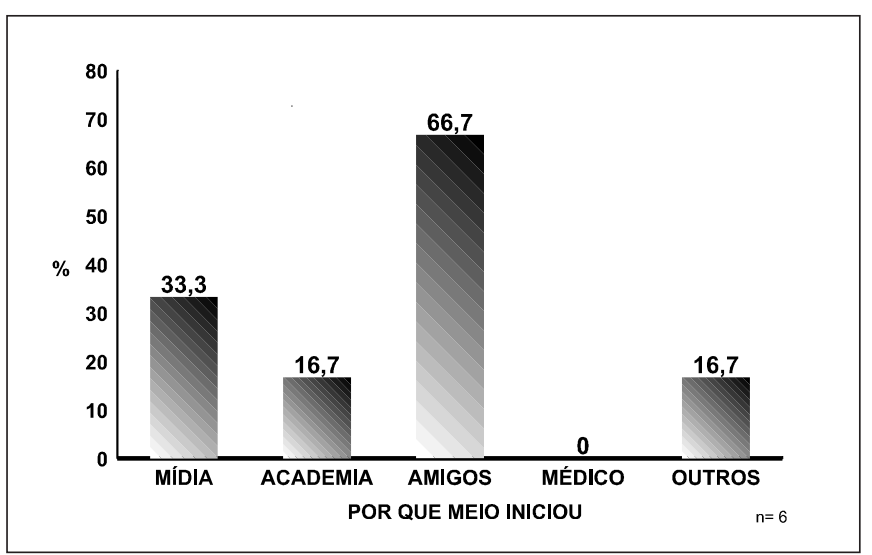

Fig. 5 - Distribuição dos praticantes de musculação elou fisioculturismo de acordo com o meio pelo qual iniciou o uso de EA

obtido em um terço dos casos. As razões principais para o uso foram o aumento da massa muscular por estética $(83,3 \%)$, melhora da auto-estima $(33,3 \%)$ e aumento da competitividade $(33,3 \%)$. Dois terços dos usuários de EA obtiveram a droga em farmácias, enquanto $16,7 \%$, com amigos ou outros meios. Quando questionados sobre o modo de aplicação do medicamento, dois terços dos entrevistados referiram usá-lo de forma injetável e metade, por via oral. O esquema de administração foi muito variável, assim como o gasto mensal.

Quando questionados se perceberam alguma diferença após iniciarem o uso de EA, apenas quatro indivíduos responderam. Um deles referiu aumento da massa muscular; outro relatou diminuição da relação músculos/gorduras após suspensão do uso; outro, o surgimento de acne; e o último, inchaço, ereções freqüentes e afirmou ser mais notado pelas mulheres. Apenas dois atletas (um terço dos usuários) demonstraram interesse na realização dos exames laboratoriais, o que inviabilizou esta etapa do projeto. 


\section{DISCUSSÃO}

No presente trabalho, dos indivíduos que relataram o uso de EA, todos eram do sexo masculino. Estudos têm mostrado que 5 a $11 \%$ dos garotos escolares nos EUA usaram EA em algum momento de sua vida ${ }^{24-27}$. Entretanto, o uso não foi restrito aos homens; 0,5 a 2,5\% das meninas também haviam, nos EUA, usado essas drogas ${ }^{28}$.

A amostra constituiu-se de indivíduos com bom nível educacional (79\% estavam, ao menos, cursando o segundo grau), mas em idade (média de 22 anos) e sexo de elevado risco para o uso de EA. Nos EUA, 40\% dos usuários de EA têm iniciado o uso antes dos 16 anos, com percentual quase equivalente de usuários da droga por via parenteral ${ }^{24,29}$.

Quase metade $(45,5 \%)$ dos entrevistados praticavam musculação e/ou fisioculturismo há mais de seis meses e 70,5\% iam à academia quatro ou mais vezes por semana. Isso constitui um tempo de exposição suficiente para que pudessem conhecer algum usuário da droga ou, até, para que pudessem tornar-se mais predispostos ao uso de EA, visto que mais de $40 \%$ dos entrevistados vinham a tomar conhecimento sobre ela através de amigos ou na própria academia.

Quando questionados sobre o uso de aditivos alimentares, 29\% posicionaram-se como usuários; os mais freqüentemente usados foram complexos vitamínicos $(51,6 \%)$ e aminoácidos $(50,5 \%)$. No entanto, quando a questão foi centrada no uso de EA, apenas $2 \%$ dos indivíduos se disseram usuários. Nos EUA, o uso geral de EA atinge índices de 5\% entre atle$\operatorname{tas}^{30-32}$. O curioso é que, embora $46 \%$ dos entrevistados afirmassem conhecer algum usuário de EA, apenas $2 \%$ dos indivíduos assumiram essa posição de usuário. Dessa forma, ou os usuários de EA são indivíduos muito "populares", ou é possível que seu número esteja subestimado. Em recente pesquisa $^{33}$, comparou-se a validade do depoimento de halterofilistas usuários de EA, por meio de um questionário, com exames de urina para a detecção da droga; 95\% dos declarados usuários tinham ao menos um EA não declarado identificado ao exame de urina, e 17,6\% dos que afirmaram não ser usuários tinham evidências objetivas da droga na urina. Dessa forma, a validade do depoimento pode ser um modo inadequado para diferenciar com acurácia usuários de não usuários de EA.

Uma grande parcela $(28,4 \%)$ dos entrevistados acredita que doses "adequadas" de EA não causem dano à saúde; ainda, 35,1\% afirmaram não conhecer os possíveis paraefeitos advindos do uso de tais drogas. Dessa forma, apesar de apenas $2 \%$ dos atletas relatarem o uso de EA, há uma quantidade razoável de indivíduos que, tanto por exposição quanto por desconhecimento, estariam em risco de, no futuro, vir a usar drogas para aperfeiçoamento físico.

Os usuários costumam combinar múltiplos esteróides em megadoses (doses 10 a 100 vezes maiores que as utilizadas para fins terapêuticos) em ciclos auto-administrados que du- ram de 4 a 12 semanas $^{6,34}$. No presente estudo, dois terços dos usuários o faziam de forma injetável, em um esquema de administração variável. Em outro estudo ${ }^{35}$, 29,2\% dos usuários de EA afirmaram compartilhar agulhas para o uso da droga; uma mesma agulha e seringa chegam a ser compartilhadas por 27 usuários consecutivos ${ }^{36}$. Dessa forma, certamente esta constitui uma questão que não vem recebendo a devida atenção em nosso meio, onde doenças como AIDS e hepatite determinam investimentos e preocupações crescentes.

Um fato de suma importância é que dois terços dos usuários adquiriram a droga em farmácias (nenhum dos usuários alegou indicação médica para o uso) e que grande parcela $(35,1 \%)$ dos atletas desconhecia seus efeitos colaterais. Isso demonstra a facilidade de obtenção da droga, amiúde por adolescentes que desconhecem seus malefícios e não têm orientações para usá-las.

O ATLAS (Adolescents Training and Learning to Avoid Steroids Program) $)^{37}$ realizou um estudo prospectivo randomizado e com posterior intervenção em atletas adolescentes, no intuito de reduzir o uso de EA. Comparado com o grupocontrole, o grupo que sofreu intervenção com palestras educativas após um ano de acompanhamento tinha elevada compreensão dos efeitos adversos dos EA, maior crença na vulnerabilidade pessoal às conseqüências adversas, menor crença nas mensagens promovidas pela mídia e melhor habilidade para recusa à oferta de EA. Outros estudos concordam em que a educação sobre o assunto pode ser efetiva na prevenção do abuso por drogas entre os atletas estudantes ${ }^{38}$ e que a nutrição e apropriado treinamento são alternativas efetivas ao uso de $\mathrm{EA}^{39}$.

Dessa forma, embora apenas $2 \%$ da amostra no presente estudo (composta predominantemente por atletas do sexo masculino com elevado nível educacional) tenham-se identificado como usuários de EA, este é um problema que necessita ser mais seriamente abordado em nosso meio. É possível que esse índice tenha sido subestimado; embora possua boa sensibilidade e especificidade ${ }^{33}$, o método aplicado pode ser inadequado para diferenciar com acurácia usuários de não usuários de EA. Uma parcela razoável de atletas desconhecia os efeitos adversos do uso da droga e acreditava que, em doses "adequadas", os EA não fossem danosos à saúde. Concluímos que a principal medida preventiva à iniciação ao uso de EA é a educação dos atletas. Deve-se, ainda, dificultar o acesso a esses medicamentos, com maior fiscalização e notificação de venda, somente efetuada com indicação e receita médicas.

\section{REFERÊNCIAS}

1. Bagatell JC, Bremner JW. Androgens in men - uses and abuses. N Engl J Med 1996;334:707.

2. Carlson MJ. Are steroids muscling in on your patients? Ohio Med 1989; 42:777. 
3. Street C, Antonio J, Cudlipp D. Androgen use by athletes: a reevaluation of the health risks. Can J Appl Physiol 1996;21:421.

4. Haupt HA. Anabolic steroids and growth hormone. Am J Sports Med 1993;21:468.

5. Snydner PJ. Clinical use of androgens. Annu Rev Med 1984;35:207.

6. Wadler GI. Drug use update. Med Clin North Am 1994;78:439.

7. Wadler GI, Hainline B: Anabolic steroids. In: Drugs and the Athlete. Philadelphia, FA Davis, 1989.

8. Krowchuk DP, Anglin TM, Goodfellow DB, Stancin T, Willians P, Zimet GD. High school athletes and the use of ergogenic aids. ASDC 1989; 143:486.

9. Rogol AD. Clinical review 31: anabolic androgenic steroids and athletes: what are the links? J Clin Endocrinol Metab 1992;74:465.

10. Rozenek R, Rahe CH, Kohl HH, Marple DN, Wilson GD, Stone MH. Physiological responses to resistance-exercise in athletes self-administering anabolic steroid. J Sports Med Phys Fit 1990;30:354.

11. Catlin D, Wrigtht J, Pope H, Lijjett M. Assessing the thresit of anabolic steroids. Phys Sportsmed 1993;21:37.

12. Ferenchick GS, Schwartz D, Ball M, et al. Androgenic-anabolic steroids abuse and platelet aggregation. Am J Med Sci 1992;303:78.

13. Freidl KE. Reappraisal of the health risks associated with the use of high doses of oral and injectable androgenic steroids. In: Lin GC, Erinoff L (eds): Anabolic Steroids Abuse. Research Monograph 102, US Department of Health and Human Services, Public Health Service, Alcohol, Drug Abuse, and Mental Health Administration, 1990.

14. Jarow JP, et al. Anabolic steroid-induced hypogonodotropic hypogonadism. Am J Sports Med 1994;18:429-31.

15. Kennedy MC, Lawrence C. Anabolic steroid abuse and cardiac health. Am J Aust 1993; 158:346.

16. Lombardo JA, Hickson RC, Lamb DR. Anabolic/androgenic steroids and growth hormone. In: Lamb DR, Williams MH (eds): Perspective in Sports Medicine. Vol. 4. Ergogenics - Enhancement of Performance in Exercise and Sports. Indianapolis: Brown \& Benchmark, 1991.

17. Pettine, KA. Association of anabolic steroids and avascular necrosis of femoral heads. Am J Sports Med 1991;19:96.

18. Strauss RH, Yesalis CE. Anabolic steroids in the athlete. Annu Rev Med 1991;42:449.

19. Wang MG, Yesalis CE, Fitzhygh EC. Desire for weight gain and potential risks of adolescent males using anabolic steroids. Percept Hot Skills 1994;78:267.

20. Lane JR, Connor JD. The influence of endogenous and exogenous sex hormones in adolescents with attention to oral contraceptives and anabolic steroids. J Adolesc Health 1994;15:630.
21. Lendstrom M, Nilsson AL, Leatzman PL, Janzon L, Dymbiny JF. Use of anabolic steroids among body builders - frequency and attitudes. J Intern Med 1990;227:407.

22. Littlepage BN, Perry HM. Misusing anabolic drugs: possibilities for future policies [editorial]. Addiction 1993;88:1469.

23. Perry PJ, et al. Illicit anabolic steroid use in athletes. Am J Sports Med 1990;18: 422.

24. Buckley W, Yesalis C, Friedl K, et al. Estimated prevalence of anabolic steroids use among male high school seniors. JAMA 1988;260:3441.

25. Johnson MD. Anabolic steroid use in adolescent athlets. Pediatr Clin North Am 1990;37:1111.

26. Johnson M, Jay M, Shoup B, et al. Adolescent steroid use in adolescent male. Pediatrics 1989;83:921.

27. Windsor R, Dumitru D. Anabolic steriods use by adolescents: survey. Med Sci Sports Exerc 1989;21:494.

28. Cowart VS. Blunting "steroid epidemic" requires alternatives, innovative education. JAMA 1990;264:1641.

29. Wright JE, Cowart VS. Performance enhancement. In: Anabolic Steroids: Altered States. Carmel, Benchmark Press, 1990.

30. Anderson WA, Albrecth MA, McKag DB, et al. A national survey of alcohol and drug use by college athletes. Phys Sportsmed 1991;19:91.

31. Anderson WA, McKeag DB. Replication of the National Study of the Substance Abuse Habits of College Student Athletes. College of Human Medicine, Michigan State University, East Lansing, 1989.

32. Anderson WA, McKeag DB. The Substance Use and Abuse Habits of College Student Athlets. College of Human Medicine, Michigan State University, East Lansing, 1985.

33. Ferenchick GS. Validity of self-reported in identifying anabolic steroid use among weighlifters. J Gen Intern Med 1996;11:554.

34. Evans NA. Gym and tonic: a profile of 100 male steroids users. Br J Sports Med 1997;31:54.

35. Melia P, Pipe A, Greenberg L. The use of anabolic-androgen steroids by Canadian students. Clin J Sports Med 1996;6:9.

36. Plates R, Temple D. The use of anabolic steroids in Wales. Welsh Committee on Drug Misuse, Cardiff, Wales, 1992.

37. Goldberg L, Elliot D, Clarke GN, McKinnon DP, Moe E, Zoerf EFL, Green C, Wolf SL, Greeffrath E, Miller DJ, Lapin A. Effects of a multidimensional anabolic steroid prevention intervention. The Adolescents Training and Learning to Avoid Steroids (ATLAS) Program. JAMA 1996; 276:1555.

38. Tricker R, Connolly D. Drug education and the college athlete: evaluation of a decision-making model. J Drug Educ 1996;26:159.

39. Elliot D, Goldberg L. Intervention and prevention of steroids use in adolescents. Am J Sports Med (Suppl) 1996;24:46. 